\title{
Vergleichende Untersuchungen zu ausgewählten Kriterien des Energiestoffwechsels in Skelettmuskulatur, Plasma und Lymphozyten bei gesunden und an Congenitaler Myofibrillärer Hypoplasie erkrankten Ferkeln
}

\author{
ANDREA BULL, PETRA ENGEL und VLADO DZAPO
}

Institut für Tierzucht und Haustiergenetik, FB09 - Agrarwissenschaften, Ökotrophologie und Umweltmanagement, Justus-Liebig-Universität Gießen, Gießen, Deutschland

\section{Zusammenfassung}

Bei an Congenitaler Myofibrillärer Hypoplasie (CMH) erkrankten Saugferkeln, deren gesunden Geschwistern sowie bei Saugferkeln aus CMH-unbetroffenen Würfen wurden die Carnitingehalte in der Skelettmuskulatur, im Blutplasma und in den Lymphozyten sowie das ATP-Bildungsvermögen der Lymphozyten- und Muskelmitochondrien und die Carnitinpalmitoyltransferase-I-(CPT I)-Aktivität bestimmt. Hierbei zeigte sich ein tendenzieller Zusammenhang zwischen dem erhöhten Anteil von Acylcarnitin am gesamten Carnitin und dem Auftreten der CMH. Gesunde Ferkel zeigten sich im ATP-Bildungsvermögen der Lymphozyten gegenüber betroffenen Tieren tendenziell überlegen. Je nach verwendetem Substrat wies das ATP-Bildungsvermögen der Muskelmitochondrien tendenzielle Unterschiede zwischen $\mathrm{CMH}$-betroffenen und gesunden Würfen auf, so dass ein Zusammenhang der Erkrankung mit dem Fettstoffwechsel, speziell der $\beta$-Oxidation, vermutet wird. Ausgeschlossen werden konnte, dass hierbei die CPT I-Aktivität eine Rolle spielt.

Schlüsselwörter: Schwein, Congenitale Myofibrilläre Hypoplasie (CMH), Grätscher, Carnitin, Mitochondrien, Carnitinpalmitoyltransferase-I (CPT I)

\section{Abstract \\ Comparative investigations of selected criteria of energy metabolism in skeletal muscle, plasma and lymphocytes in healthy and in piglets with congenital myofibrillar hypoplasia}

Carnitine contents of skeletal muscle, plasma and lymphocytes, the capacity of ATPsynthesis in muscle mitochondria and lymphocytes, and the enzyme activity of carnitine palmitoyltransferase I (CPT I) in muscle mitochondria were determined in piglets with congenital myofibrillar hypoplasia (CMH) in comparision to their clinical healthy siblings and piglets from $\mathrm{CMH}$-free litters. Tendentious differences in the relation of acylcarnitine to free carnitine in healthy piglets and piglets suffering from $\mathrm{CMH}$ could be detected. Healthy piglets showed in tendency superiority in the ATP-production of their lymphocytes compared with diseased animals. Depending on the applied substrate, muscle mitochondria showed tendentially differences in the ATP-production between $\mathrm{CMH}$-affected and healthy litters, so that a connection between $\mathrm{CMH}$ and the lipid 
metabolism, particular the $\beta$-oxidation, is supposed. Differences in the carnitine palmitoyltransferase l-activity as a possible cause for $\mathrm{CMH}$ could be excluded.

Keywords: pig, congenital myofibrillar hypoplasia $(\mathrm{CMH})$, splayleg, carnitine, mitochondria, carnitine palmitoyltransferase I (CPT I)

\section{Einführung}

Der Begriff »Congenitale Myofibrilläre Hypoplasie« (CMH) bezeichnet eine Störung, die angeboren, d.h. durch Vererbung (SCHWARZ 2003, VOGT et al. 1984) oder intrauterin durch eine auf die Sau einwirkende Noxe (BÖLCSKEl et al. 1996, VANYI et al. 1994, JIRMANOVA und LOJDA 1985, TUCEK et al. 1985, DUCATELLE et al. 1986, SELLIER et al. 1999) erworben wird, und einen bisher nur klinisch und histopathologisch in der Skelettmuskulatur darstellbaren Befund beschreibt. Charakteristischerweise ist stets die Muskelgruppe der Adduktoren der Hintergliedmaßen betroffen, seltener manifestiert sich die Erkrankung gleichzeitig an den Vordergliedmaßen, woraus eine schwere Behinderung der Fortbewegung resultiert. In der Umgangssprache werden erkrankte Ferkel als »Grätscher« oder »Spreizer« bezeichnet, im englischen Sprachraum spricht man von der Erkrankung als "Splayleg«. Die Insuffizienz der Muskulatur an sich ist nicht tödlich, und bereits innerhalb der ersten Lebenswoche stellt sich die volle Funktionstüchtigkeit ein, aber bis dahin droht den Ferkeln der Erdrückungstod durch die Muttersau, und eine Vielzahl verhungern oder sterben an Unterkühlung (WARD 1978).

Laut STIGLER et al. (1992) sind bis zu 0,7\% der neugeborenen Ferkel betroffen. Eine Auswertung der Daten der Besamungsstation Ascheberg in Nordrhein-Westfalen durch FREITAG und KLEIN (2003) ergab, dass insgesamt 2,9\% der Ferkel und 20,2\% der Würfe durch das Vorkommen von Anomalien auffielen. Fast die Hälfte der Defekte (49\%) betraf dabei die Spreizbeinigkeit.

Immer noch besteht eine gewisse Uneinigkeit in Bezug auf die Erblichkeit der $\mathrm{CMH}$. Man vermutet eine multifaktorielle Ätiologie, basierend auf einer genetischen Komponente und umweltbedingten Auslösern. Die Vererbung erfolgt wahrscheinlich polygen, mit einem Heritabilitätskoeffizienten (Halbgeschwisteranalyse) von 0,47 (SELLIER und OLLIVER 1982). Der Defekt konnte molekularbiologisch bisher nicht lokalisiert werden, möglicherweise liegen polymorphe, regulatorische Elemente auf den Chromosomen 5 und 11 (MAAK et al. 2001, 2003, 2005, 2007, SCHWARZ 2003).

Neben der Unklarheit über die genauen Pathogenitätsmechanismen der $\mathrm{CMH}$ sind auch die Details der Ausprägung biochemischer Abweichungen gegenüber gesunden Tieren weitgehend unbekannt. In der vorliegenden Untersuchung sollen die Unterschiede gesunder und kranker Ferkel in einem Teilbereich des Energiestoffwechsels betrachtet werden. Carnitin fördert die Fettverbrennung durch die Einschleusung von Fettsäuren in die Mitochondrienmatrix (FRIEDMANN und FRAENKEL 1955, FRITZ 1955). Durch den Abbau der Fettsäuren zu Acetyl-CoA-Einheiten in der ß-Oxidation und der sich anschließenden Verwertung des Acetyl-CoA im Citratzyklus erfolgt die energetische Nutzung. Da die Fettsäurenverwertung für die Energieversorgung des Muskels und damit für seine Funktionsfähigkeit eine bedeutende Rolle spielt, wurden die Gehalte des dafür essentiellen Carnitins und die Aktivität des zum Carnitinsystem gehörigen Enzyms 
Carnitinpalmitoyltransferase I (CPTI) gemessen, sowie die mitochondriale ATPSynthesekapazität in Muskulatur und Lymphozyten untersucht und verglichen. Dies erfolgte außerdem in Hinblick auf die Fragestellung, ob sich die bei CMH eventuell vorhandenen biochemischen Abweichungen in der Muskulatur ebenfalls in den Blutzellen nachweisen lassen, auf die unter Umständen ein besserer diagnostischer Zugriff zu nehmen wäre.

\section{Material und Methoden}

\section{Untersuchte Tiere}

Die untersuchten Ferkel wurden von der Lehr- und Forschungsstation "Oberer Hardthof" der Justus-Liebig-Universität Gießen zur Verfügung gestellt. Für die Untersuchungen wurden sechs Gruppen mit jeweils drei Tieren gebildet. Diese setzten sich zusammen aus einem Ferkel mit den klinischen Symptomen der CMH (»Grätscher«), einem weiteren gesunden Vollgeschwistertier (»Geschwister«), sowie einem möglichst gleichaltrigen Ferkel aus einem unbetroffenen Wurf (»Kontrolle«). Die klinische Diagnose »CMH« erfolgte in Anlehnung an die von SCHNAPPERELLE und KOCH (1980) vorgeschlagenen diagnostischen Kriterien (Schweregrad II bis IV). Hierbei mussten die Bewegungsstörungen sofort nach der Geburt aufgetreten und auch noch 24 Stunden post partum vorhanden sein. Des Weiteren waren mindestens die Hintergliedmaßen mit dem charakteristischen Ausgrätschen betroffen und die Ferkel zeigten ein ungetrübtes Sensorium, sowie auch keine Hinweise auf infektiöse Erkrankungen oder Traumata bestanden. Die Muttersauen wurden nicht hormonell behandelt, die Geburten erfolgten spontan zwischen dem 114. und 116. Trächtigkeitstag und wurden nicht durch Prostaglandine eingeleitet. Ebenso unterblieb während der Trächtigkeit jede Behandlung der Sauen mit Cortikoiden. Anpaarungen mit der Deutschen Landrasse (DL) und dem Deutschen Edelschwein (DE) wurden als sogenannte Mutterlinie zusammengefasst, die Vaterlinie wurde durch die Rasse Piétrain (Pi) repräsentiert. Tabelle 1 gibt eine Übersicht über Alter, Gewicht und Anzahl der untersuchten Tiere, sowie deren Verteilung in den Merkmalen Vaterrasse und Geschlecht.

Tabelle 1

Anzahl $(n)$ der untersuchten Tiere mit unterschiedlichen CMH-Status, Mittelwerte und Standardabweichungen in den Merkmalen Alter und Gewicht, sowie die Anzahl der Ferkel in Bezug auf ihre Geschlechts- und Vaterrassenzugehörigkeit

Number of investigated piglets with different $\mathrm{CMH}$-status, mean and standard deviation in the parameters age and weight, and number of piglets in the different categories of father breed and sex

\begin{tabular}{lccccc}
\hline CMH-Status & $n$ & Alter, Tage & Gewicht, kg & Geschlecht, $\mathrm{m} / \mathrm{w}$ & $\begin{array}{c}\text { Vaterrasse, } \\
\text { DL+DE/Pi }\end{array}$ \\
\hline Ferkel (gesamt) & 18 & $3,67 \pm 2,28$ & $1,67 \pm 0,40$ & $12 / 6$ & $8 / 10$ \\
Grätscher & 6 & $3,17 \pm 1,94$ & $1,45 \pm 0,30$ & $5 / 1$ & $2 / 4$ \\
Geschwister & 6 & $3,17 \pm 1,94$ & $1,75 \pm 0,43$ & $2 / 4$ & $2 / 4$ \\
Kontrolle & 6 & $4,67 \pm 2,88$ & $1,80 \pm 0,43$ & $5 / 1$ & $4 / 2$ \\
\hline
\end{tabular}




\section{Aufbereitung der Muskelproben und Mitochondrienisolation}

Die Skelettmuskelproben entstammten der Adduktorenmuskulatur der Hintergliedmaßen ( $M$. adduktor). Die Mitochondrienisolierung erfolgte nach der modifizierten Methode von MERSMANN et al. (1972). Mittels eines Potter-Elvehjem-Glas-TeflonHomogenisators wurden die Zellkompartimente schonend eröffnet und die freigesetzten Mitochondrien durch anschließende Differentialzentrifugation gewonnen. Zur Aufnahme und Erhaltung diente das Isolationsmedium nach SCHMIDT und HERPIN (1998) aus $220 \mathrm{mM}$ Mannitol, $70 \mathrm{mM}$ Saccharose, $2 \mathrm{mM}$ HEPES und 0,1 mM EDTA. Die Proteinbestimmung in der Suspension erfolgte nach der Methode von LOWRY et al. (1951).

\section{Aufbereitung der Blutproben zur Plasmagewinnung und Lymphozytenisolation}

Von den Ferkeln wurde $K^{+} E D T A-B l u t ~ g e w o n n e n$. Für die Plasmagewinnung wurde die Probe $10 \mathrm{~min}$ bei $500 \mathrm{~g}$ zentrifugiert. Zur Lymphozytenisolation wurden die Proben mit Dextran (5\% in PBS) durchmischt und 30 min lang in einem $37^{\circ} \mathrm{C}$ warmen Wasserbad inkubiert. Nach dem Absinken der roten Blutkörperchen wurde das Dextranplasma abgenommen und daraus durch Dichtegradientenzentrifugation unter Verwendung von Ficoll Paque Plus die Lymphozyten isoliert. Das Lymphozytenpellet wurde danach in eiskaltem Isolationsmedium nach CARPENTIERI und SORDAHL (1980), bestehend aus 0,25 M Saccharose, $5 \mathrm{mM}$ TRIS-HCl, 5 mM EGTA und 0,5\% Bovinem Serumalbumin resuspendiert. Der als Bezugsgröße herangezogene Proteingehalt in der Lymphozytensuspension wurde ebenfalls nach der Methode von LOWRY et al. (1951) bestimmt.

\section{Bestimmung des ATP-Bildungsvermögens}

Die Messung des ATP-Gehaltes erfolgte als photometrischer Nachweis mit 3-PhosphoglyceratKinase mittels einer modifizierten Methode nach BÜCHER (BERGMEYER et al. 1974).

Aus der Lymphozyten- bzw. Muskelmitochondriensuspension wurde pro Versuchsansatz eine definierte Menge mit bekanntem Proteingehalt entnommen, diese zu einem Inkubationsmedium hinzugegeben $(0,25 \mathrm{mM}$ phosphatgepufferte Saccharoselösung, wie beschrieben bei CARPENTIERI und SORDAHL [1980] für die Lymphozyten, und bei SCHMIDT und HERPIN [1998] für die Muskelmitochondrien), und dann im SchüttlerWasserbad bei $30^{\circ} \mathrm{C}$ für 5 min inkubiert. Danach wurden entweder Succinat $(7,5$ $\mu \mathrm{mol} /$ Ansatz) oder Palmitoylcarnitin $(0,2 \mu \mathrm{mol} /$ Ansatz) als Substrate hinzugefügt, und die Inkubation weitere $5 \mathrm{~min}$ lang fortgesetzt. Zuletzt wurde ADP hinzugegeben $(0,76$ $\mu \mathrm{mol} /$ Ansatz bei den Lymphozyten und 1,9 $\mu \mathrm{mol} /$ Ansatz bei den Muskelmitochondrien), welches die Hauptreaktion im Status 3 der mitochondrialen Atmung startet. Die ATPSynthese wurde nach 5 min durch Herunterkühlen des Probengefäßes in flüssigem Stickstoff abgestoppt. Zur Aufbereitung für die photometrische Messung wurden die Proben enteiweißt und mit Ultraschall homogenisiert.

\section{Carnitinbestimmung}

In der von der Carnitinacetyltransferase katalysierten Reaktion überträgt Tritiummarkiertes Acetyl-CoA den markierten Acetylrest auf das freie Carnitin (KERNER und BIEBER 1983, McGARRY und FOSTER 1985, GUSTAVSEN 2000). Das überschüssige $\left[{ }^{3} \mathrm{H}\right]$-Acetyl-CoA wird 
durch einen Anionenaustauscher gebunden, den $\left[{ }^{3} \mathrm{H}\right]$-Acetylcarnitin passieren kann. Dessen Gehalt kann im Vergleich mit einem Carnitinstandard mittels Flüssigkeitszintillationszählung bestimmt werden.

Für die Bestimmung des Gesamtcarnitins mussten die Carnitinester zuerst mit Hilfe einer 0,2 N Kaliumhydroxydlösung einer alkalischen Hydrolyse unterzogen werden, um sie in freies Carnitin zu überführen. Die Gehalte an Acylcarnitin wurden aus der Differenz rechnerisch ermittelt. Die Lymphozytensuspension wurde zuvor $20 \mathrm{~min}$ lang im Ultraschallbad ( $360 \mathrm{~W}$ ) beschallt, danach wurden die Proben auf $98^{\circ} \mathrm{C}$ erhitzt, um unspezifische Reaktionen zu verhindern.

Die Muskelprobe wurde zunächst homogenisiert und lyophilisiert, aus dem Lyophilisat unter Ultraschalleinwirkung ein wässriger Extrakt hergestellt, und der Überstand zur Carnitinmessung herangezogen.

Bestimmung der Enzymaktivität der Carnitinpalmitoyltransferase CPT I

(EC 2.3.1.21) in den Mitochondrien aus dem M. adduktor

Die Bestimmung erfolgte als sogenannter Isotopen-Exchange-Assay mittels einer Methode nach NORUM (1964), modifiziert durch BREMER (1981) und SCHMIDT und HERPIN (1998).

\section{Statistische Auswertung}

Die Auswertung der Daten erfolgte mit dem Programmpaket SPSS (Version 15.0). Anhand dieser Software erfolgte die Berechnung von Korrelationen nach Pearson, von linearen Regressionen und Varianzanalysen. CMH-Gesundheitsstatus, Vaterrasse, Geschlecht, Carnitingehalte im Blutplasma der Muttersau und/oder das Alter der beprobten Ferkel wurden in linearen Modellen als Effekte und Kovariablen auf Carnitingehalte bzw. -anteile in der Muskeltrockensubstanz, im Plasma und in Lymphozyten, dem produzierten ATP in Muskelmitochondrien und Lymphozyten, sowie der CPT I-Aktivität in Muskelmitochondrien herangezogen.

\section{Ergebnisse}

\section{Carnitingehalte in der Muskeltrockensubstanz}

Unter Berücksichtigung der Einflussfaktoren CMH-Gesundheitsstatus, Vaterrasse, Geschlecht und Alter konnte der Einfluss des Alters auf den Carnitingehalt in der Muskeltrockensubstanz statistisch nachgewiesen werden $(P=0,023)$. Je älter das Saugferkel war, umso höher fielen die Carnitingehalte in der Muskeltrockenmasse aus $(b=0,409 \mu \mathrm{mol}$ Gesamtcarnitin/g Muskeltrockenmasse). Die Vaterrasse und das Geschlecht zeigten tendenzielle Einflüsse auf die verschiedenen Carnitinfraktionen, die aber statistisch nicht abgesichert werden konnten. Der Einfluss des unterschiedlichen CMH-Status der Ferkel auf den Gesamtcarnitingehalt bewegte sich an der Signifikanzgrenze $(P=0,074)$, wobei die Gruppe der Grätscher im Vergleich zu den klinisch gesunden Wurfgeschwistern und den Kontrollferkeln die höchsten Gesamtcarnitinwerte in der Muskeltrockensubstanz aufwiesen (Tabelle 2). 
Auch bei den Acylcarnitingehalten war diese Rangfolge zu beobachten, während beim freien Carnitin für die Gruppe der Grätscher die niedrigsten Randmittelwerte im Vergleich zu den Kontrolltieren und Geschwistern berrechnet wurden. Weder die berücksichtigten Einflussfaktoren noch die beobachteten Unterschiede zwischen Ferkeln mit unterschiedlichem CMH-Status konnten für die Gehalte an freiem Carnitin und an Acylcarnitin in der Muskeltrockensubstanz statistisch abgesichert werden.

Tabelle 2

Randmittelwerte der Carnitingehalte in der Muskeltrockensubstanz von Saugferkeln mit unterschiedlichem CMH-Gesundheitsstatus unter Angabe der Mittelwerte der jeweiligen Carnitinfraktionsgehalte

Least square mean of carnitine in dried matter of skeletal muscle of piglets for the influence factor $\mathrm{CMH}$ status with information about the mean of the respective carnitine fraction contents

\begin{tabular}{lccc}
\hline Carnitinfraktion & Gesamtcarnitin, $\mu \mathrm{mol} / \mathrm{g}$ & freies Carnitin, $\mu \mathrm{mol} / \mathrm{g}$ & Acylcarnitin, $\mu \mathrm{mol} / \mathrm{g}$ \\
\hline Mittelwert der Carnitinfraktion & 4,089 & 3,309 & 0,780 \\
CMH-Status: Grätscher & $4,980^{\mathrm{a}}$ & 3,474 & 1,506 \\
Geschwister & $3,535^{\mathrm{a}}$ & 2,813 & 0,722 \\
Kontrolle & $3,752^{\mathrm{a}}$ & 3,640 & 0,112 \\
\hline
\end{tabular}

aSignifikanz der Mittelwertunterschiede im Gesamtcarnitingehalt von Ferkeln unterschiedlichen CMH-Gesundheitsstatus: $\mu_{\text {Grätscher }} / \mu_{\text {Geschwister }}: P=0,034, \mu_{\text {Grätscher }} / \mu_{\text {Kontrolle: }}: P=0,102, \mu_{\text {Kontrolle }} / \mu_{\text {Geschwister: }}: P=0,76$

Der Anteil von freiem Carnitin und Acylcarnitin am gesamten Carnitin in der Muskeltrockensubstanz betrug $82 \%$ bzw. 18\%. Eine Veränderung der prozentualen Anteile von freiem Carnitin und Acylcarnitin am Gesamtcarnitin war zwischen $\mathrm{CMH}$ betroffenen und freien Tieren zu beobachten. Hierbei stieg der prozentuale Anteil des Acylcarnitins von den Kontrolltieren (2\%) über die Geschwister (18\%) zu den Grätschern (34\%) hin an. Der Anteil an freiem Carnitin bzw. der Acylcarnitinanteil der Grätscher unterschied sich signifikant $(P<0,05)$ von dem der Kontrolltiere.

Wurde als weiterer möglicher Einflussfaktor der Gehalt der jeweiligen Carnitinfraktion im Plasma der Muttersau berücksichtigt, konnte kein signifikanter Einfluss auf die Carnitingehalte im Muskelgewebe der Nachkommen beobachtet werden.

\section{Carnitingehalte im Blutplasma}

Während die Carnitingehalte im Plasma der Muttersau keinen Einfluss auf die Gehalte in der Muskulatur der Ferkel nahmen, hatte der Gehalt der jeweiligen Carnitinfraktion im Blutplasma der Muttersau einen positiv gerichteten Einfluss auf den entsprechenden Gehalt der Carnitinfraktion im Plasma des Ferkels. Hierbei verursachte der Acylcarnitingehalt im Plasma der Muttersau die größten Veränderungen im Vergleich zu den Gehalten an gesamten und freien Carnitin (Abbildung 1).

Auch unter zusätzlicher Berücksichtigung der Einflussfaktoren Vaterrasse, Geschlecht, $\mathrm{CMH}-$ Status und Alter des Ferkels konnte lediglich der Einfluss der Carnitinfraktion im Blutplasma der Muttersau auf den entsprechenden Carnitinfraktionsgehalt im Ferkelblutplasma statistisch abgesichert werden $(P<0,01$, Tabelle 3$)$. Der Einfluss des $\mathrm{CMH}-$ Gesundheitstatus auf den Carnitingehalt im Plasma der Ferkel bewegte sich je nach entsprechendem maternalen Plasmacarnitinfraktionsgehalt bei Irrtumswahrscheinlichkeiten von $8 \%$ bis $12 \%$. Grätscher unterschieden sich in ihrem niedrigen Gehalt an gesamtem Carnitin im Blutplasma von ihren Geschwistern $(P=0,044)$. Geschwister und 
Kontrolltiere unterschieden sich nicht signifikant in Bezug auf die Carnitinfraktionsgehalte im Plasma. Tendenziell wiesen Grätscher ebenfalls die geringsten absoluten Gehalte an freiem Carnitin und an Acylcarnitin auf.

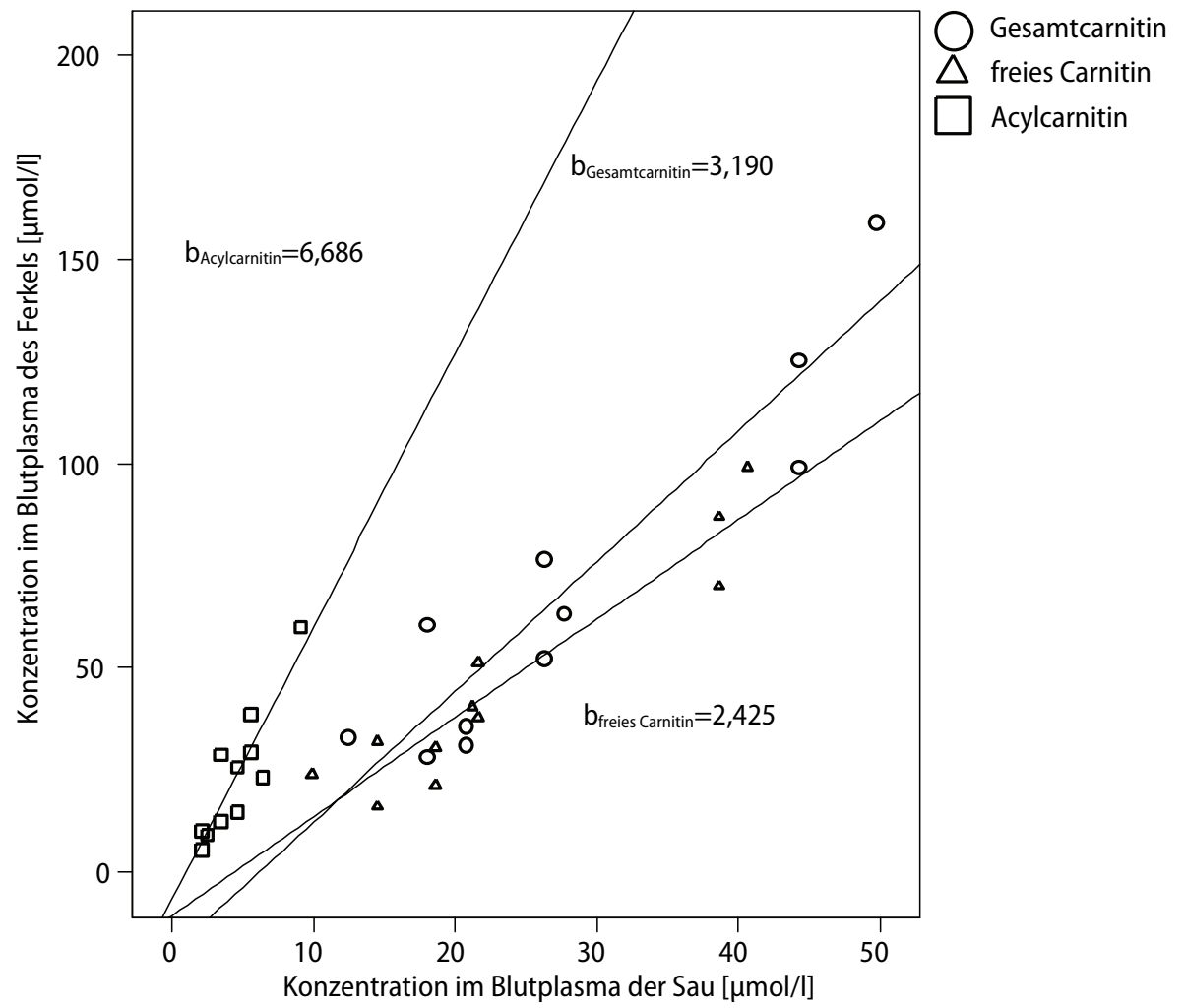

Abbildung 1

Streudiagramm mit jeweiliger Regressionsgeraden und -koeffizienten für die Abhängigkeit der Carnitingehalte im Blutplasma der Ferkel von den jeweiligen Carnitingehalten im Blutplasma der Muttersauen

Scatterplot with linear regression and coefficient showing the dependence of the carnitine concentration in the plasma of piglets from the respective carnitine fraction concentration in the plasma of the mother sow

Tabelle 3

Randmittelwerte der Carnitingehalte im Blutplasma von Saugferkeln mit unterschiedlichem $\mathrm{CMH}$ Gesundheitsstatus unter Angabe der Mittelwerte der jeweiligen Carnitinfraktionsgehalte

Least square mean of carnitine concentration in the plasma from piglets for the influence factor $\mathrm{CMH}$-status with information about the mean of the respective carnitine fraction contents

\begin{tabular}{cccc}
\hline Carnitinfraktion & gesamtes Carnitin, $\mu \mathrm{mol} / \mathrm{l}$ & freies Carnitin, $\mu \mathrm{mol} / \mathrm{l}$ & Acylcarnitin, $\mu \mathrm{mol} / \mathrm{l}$ \\
\hline Mittelwert der Carnitinfraktion & 65,656 & 44,117 & 22,232 \\
CMH-Status: Grätscher & $47,939^{\mathrm{a}}$ & $34,027^{\mathrm{b}}$ & $17,083^{\mathrm{c}}$ \\
Geschwister & $74,275^{\mathrm{a}}$ & $47,895^{\mathrm{b}}$ & $27,788^{\mathrm{c}}$ \\
Kontrolle & $74,755^{\mathrm{a}}$ & $50,428^{\mathrm{b}}$ & $21,826^{\mathrm{c}}$ \\
\hline
\end{tabular}

Signifikanz der Randmittelwertunterschiede von Ferkeln unterschiedlichen $\mathrm{CMH}$-Gesundheitsstatus im ${ }^{\mathrm{a}} \mathrm{Gesamt}$ carnitingehalt: $\mu_{\text {Grätscher }} / \mu_{\text {Geschwister }} P=0,04, \mu_{\text {Grätscher }} / \mu_{\text {Kontrolle: }} P=0,07, \mu_{\text {Kontrolle }} / \mu_{\text {Geschwister: }} P=0,97$ b Gehalt an freiem Carnitin: $\mu_{\text {Grätscher }} / \mu_{\text {Geschwister: }} P=0,07, \quad \mu_{\text {Grätscher }} / \mu_{\text {Kontrolle: }} P=0,08, \quad \mu_{\text {Kontrolle }} / \mu_{\text {Geschwister: }} P=0,71 \quad{ }^{c}$ Aylcarnitingehalt: $\mu_{\text {Grätscher }} / \mu_{\text {Geschwister }}: P=0,06, \mu_{\text {Grätscher }} / \mu_{\text {Kontrolle }}: P=0,39, \mu_{\text {Kontrolle }} / \mu_{\text {Geschwister: }} P=0,29$ 
Der Unterschied zwischen $\mathrm{CMH}$-betroffenen Ferkeln im Vergleich zu gesunden Tieren zeigte sich stärker in den Anteilen von Acylcarnitin und freiem Carnitin am Gesamtcarnitin im Plasma. So betrug der durchschnittliche Anteil des freien Carnitins am Gesamtcarnitin unter Berücksichtigung der Einflussfaktoren Vaterrasse, Geschlecht, CMH-Status und Alter des Ferkels $68,4 \%$. Bei Betrachtung der Zusammensetzung des gesamten Carnitins, konnte berechnet werden, dass der Anteil des Acylcarnitins im Plasma bei den CMHbetroffenen Ferkeln niedriger ausfiel (26,9\%) im Vergleich zu den gesunden Tieren (Geschwister: 36\%, Kontrolle: 31,8\%). Diese Beobachtung konnte jedoch statistisch nicht abgesichert werden.

Die Carnitingehalte in der Muskeltrockensubstanz der Ferkel waren zu denen im Blutplasmaspiegel desselben Tieres nur für das freie Carnitin miteinander signifikant korreliert $(r=0,683, P<0,05)$.

\section{Carnitingehalte in den Lymphozyten}

Unter Berücksichtigung der Einflussfaktoren Vaterrasse, Geschlecht, CMH-Status und Alter des Ferkels konnte für die Gruppe der Grätscher die höchsten Gesamtcarnitingehalte in den Lymphozyten gemessen werden, gefolgt von den Wurfgeschwistern. Die geringsten Gehalte waren bei den Ferkeln aus gesunden Würfen zu verzeichnen (Abbildung 2).

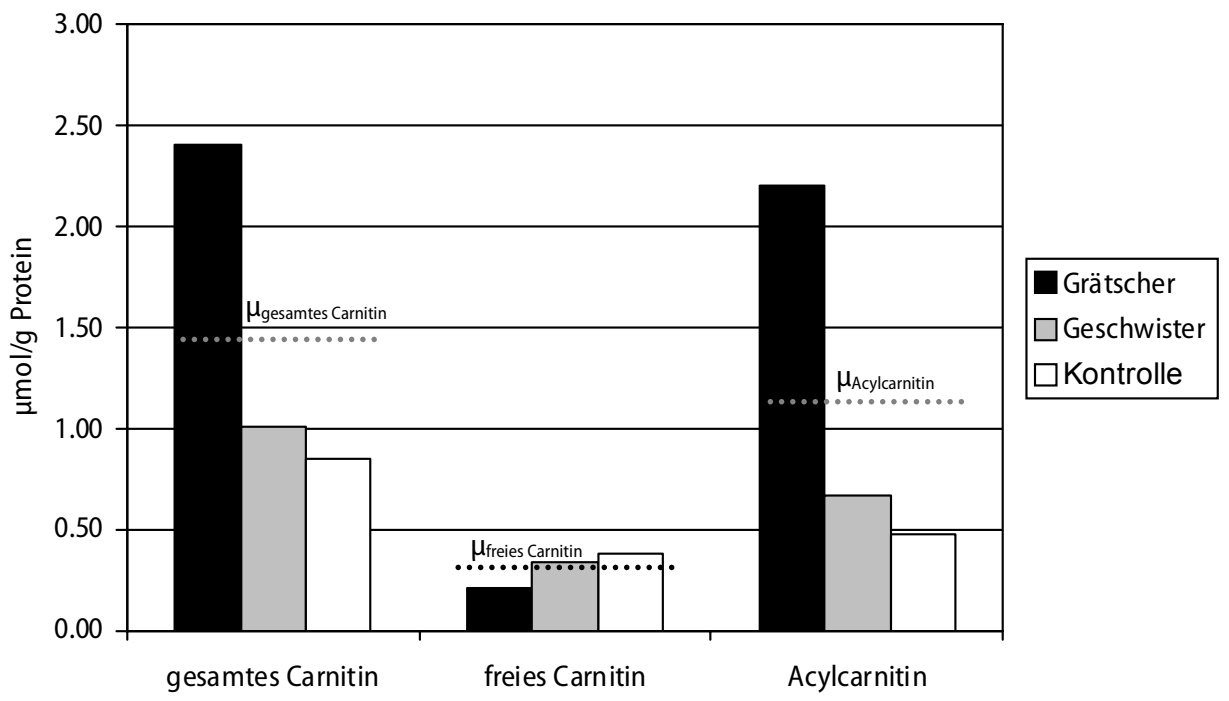

Abbildung 2

Randmittelwerte der Carnitingehalte in Lymphozyten von Saugferkeln mit unterschiedlichem $\mathrm{CMH}$ Gesundheitsstatus unter Angabe der Mittelwerte der jeweiligen Carntinfraktionsgehalte $(1,42 \mu \mathrm{mol}$ gesamtes Carnitin/g, 0,30 $\mu \mathrm{mol}$ freies Carnitin/g, 1,12 $\mu \mathrm{mol}$ Acylcarnitin/g)

Least square mean of carnitine concentrations in lymphocytes of piglets with different $\mathrm{CMH}$-status including information about the mean of the respective carnitine fraction contents $(1,42 \mu \mathrm{mol}$ total carnitine $/ g, 0,30$ $\mu \mathrm{mol}$ free carnitine/g, 1,12 $\mu \mathrm{mol}$ acylcarnitine/g)

Die hohen Gesamtcarnitingehalte in den Lymphozyten wurden durch sehr hohe Acylcarnitingehalte (durchschnittlich $74,5 \%$ des Gesamtcarnitins) bedingt, während das freie Carnitin nur einen geringen Anteil ausmachte. Beim Vergleich des relativen Acylcarnitingehaltes zwischen den Ferkeln mit unterschiedlichem CMH-Gesundheitsstatus 
konnte ein Unterschied für Grätscher mit einem durchschnittlichen Gehalt von 89\% Acylcarnitin am gesamten Carnitin im Lymphozytenprotein gegenüber den Geschwisterund den Kontrollferkeln mit $63 \%$ bzw. $71 \%$ beobachtet werden. Diese Unterschiede konnten trotz der hohen erklärten Varianz des verwendeten Modells (71,2\%-98,4\%) sowohl für den $\mathrm{CMH}-$ Gesundheitsstatus als auch für die anderen berücksichtigten Einflussfaktoren nicht statistisch abgesichert werden.

\section{Korrelationen der Carnitingehalte in Lymphozyten und Muskulatur}

Die Gehalte an freiem Carnitin und Gesamtcarnitin in den Lymphozyten waren positiv korreliert mit den entsprechenden Gehalten in der Muskeltrockensubstanz der Ferkel $\left(r_{\text {freies }}\right.$ Carnitin $\left.=0,852, P<0,05 ; r_{\text {Gesamtcannitin }}=0,716, P<0,01\right)$. Im Bereich des Acylcarnitins existierte lediglich eine geringe negativ gerichtete nicht signifikante Beziehung $(r=-0,369)$ zwischen dem Gehalt in den Lymphozyten und dem in der Muskeltrockensubstanz.

\section{Unterschiede in der ATP-Synthese der Lymphozyten zwischen Ferkeln mit/ohne CMH}

Desweiteren wurde untersucht, ob sich Ferkel mit CMH von ihren Geschwistern oder Ferkeln aus nicht betroffenen Würfen hinsichtlich der ATP-Bildungsfähigkeit der Lymphozyten bei Verwendung der Substrate Succinat und Palmitoylcarnitin unterscheiden.

Es konnten in keinem Versuchsansatz Signifikanzen für die im Modell berücksichtigten Einflussfaktoren (Vaterrasse, Geschlecht und CMH-Status) auf die ATP-Produktion der Lymphozyten nachgewiesen werden. Dennoch zeigte sich bei Zugabe von ADP für die beiden verwendeten Substrate Succinat und Palmitoylcarnitin die Tendenz einer höheren ATP-Produktion der Lymphozyten von Ferkeln, die aus einem unbelasteten Wurf stammten, im Vergleich zu Ferkeln, die selber unter $\mathrm{CMH}$ litten oder deren Wurfgeschwister (Abbildung 3).

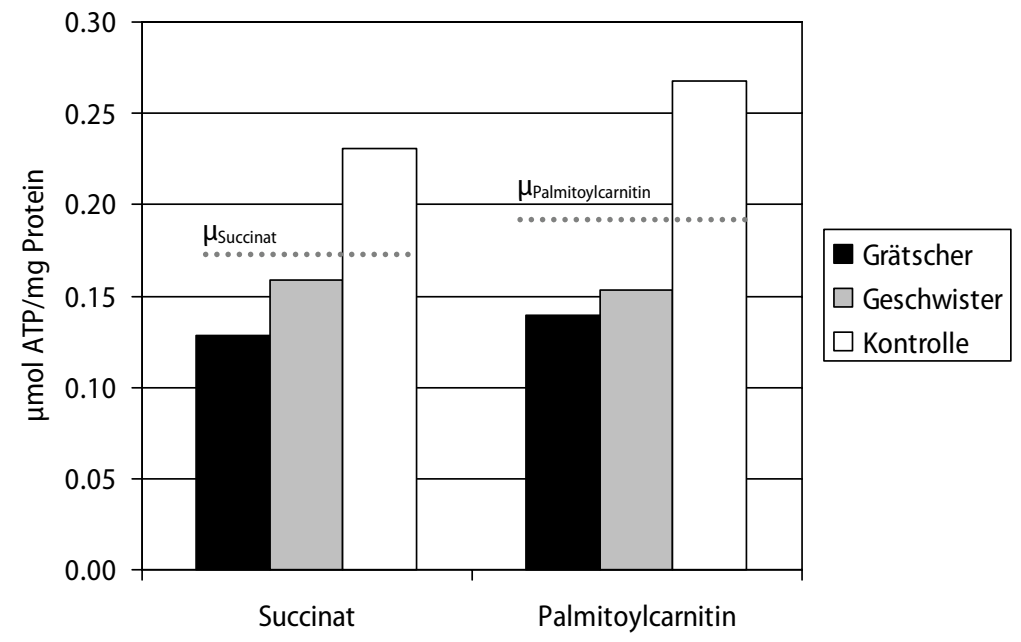

Abbildung 3

Randmittelwerte der ATP-Produktion der Lymphozyten von Saugferkeln mit unterschiedlichem CMHStatus unter Angabe der Mittelwerte des jeweiligen Substrates (Succinat: 0,17 $\mu \mathrm{mol}$ ATP/mg, Palmitoylcarnitin: 0,19 $\mu \mathrm{mol}$ ATP/mg)

Least square mean of ATP-production of lymphocytes from piglets of different $\mathrm{CMH}$-status with information about the mean of the respective substrate (succinate: $0,17 \mu \mathrm{mol}$ ATP/mg, palmitoylcarnitine: $0,19 \mu \mathrm{mol}$ ATP/mg) 
Unterschiede in der ATP-Synthese der Skelettmuskelmitochondrien mit den Substraten Succinat oder Palmitoylcarnitin und ADP zwischen Ferkeln mit und ohne CMH

Unter Berücksichtigung der Einflussfaktoren Vaterrasse, Geschlecht und CMH-Status produzierten die Muskelmitochondrien von Ferkeln aus CMH betroffenen Würfen bei Verwendung des Substrates Succinat mehr ATP als die der unbelasteten Kontrolltiere (Abbildung 4). Hierbei konnte für die Geschwister der Grätscher die höchste mitochondriale ATP-Produktion gemessen werden. Letzteres war auch unter Verwendung des Substrates Palmitoylcarnitin der Fall, wohingegen die Grätscher den geringsten Anteil an der ATP-Produktion zeigten. Diese Beobachtungen als auch Einflüsse durch die im Modell berücksichtigten Faktoren konnten statistisch nicht abgesichert werden.

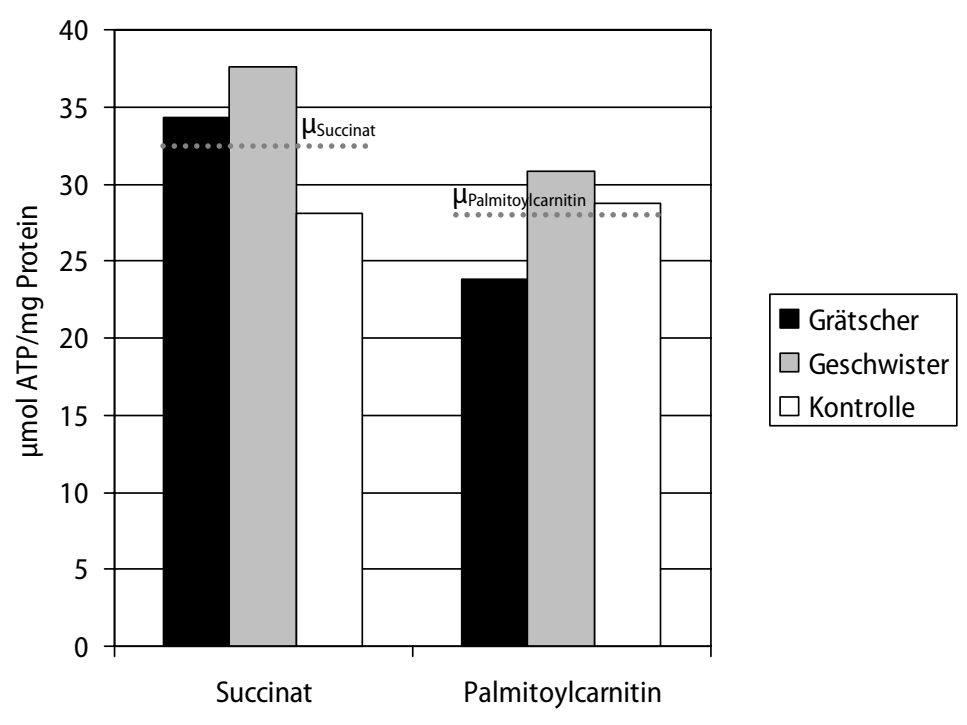

Abbildung 4

Randmittelwerte der ATP-Produktion der Muskelmitochondrien von Saugferkeln mit unterschiedlichem CMH-Status unter Angabe der Mittelwerte des jeweiligen Substrates (Succinat: 33,4 $\mu \mathrm{mol}$ ATP/mg, Palmitoylcarnitin: $27,8 \mu \mathrm{mol}$ ATP/mg)

Least square mean of ATP-production of muscle mitochondria from piglets with different CMH-status including information about the mean of the respective substrate (succinate: $33,4 \mu \mathrm{mol} A T P / \mathrm{mg}$, palmitoylcarnitine: $27,8 \mu \mathrm{mol}$ ATP/mg)

\section{Einfluss des CMH-Syndroms auf die Aktivität der CPT I in Muskelmitochondrien}

In Abbildung 5 sind die berücksichtigten Einflussfaktoren auf die CPT I-Aktivität der Muskelmitochondrien dargestellt. Hierbei kamen vor allem Rassenunterschiede zum Tragen. Ferkel, deren Väter der Rasse Piétrain angehörten, wiesen eine höhere Enzymaktivität auf als Ferkel, die von Ebern der Rassen DL oder DE abstammten. Der Rasseneinfluss bewegte sich statistisch gesehen an der Signifikanzgrenze $(P=0,075)$.

Das Geschlecht des Ferkels spielte für die Höhe der CPTI-Aktivität keine Rolle. Grätscherferkel besaßen tendenziell eine etwas höhere CPTI-Aktivität als ihre gesunden Wurfgeschwister und die Kontrollen, diese Beobachtung konnte jedoch statistisch nicht abgesichert werden. 


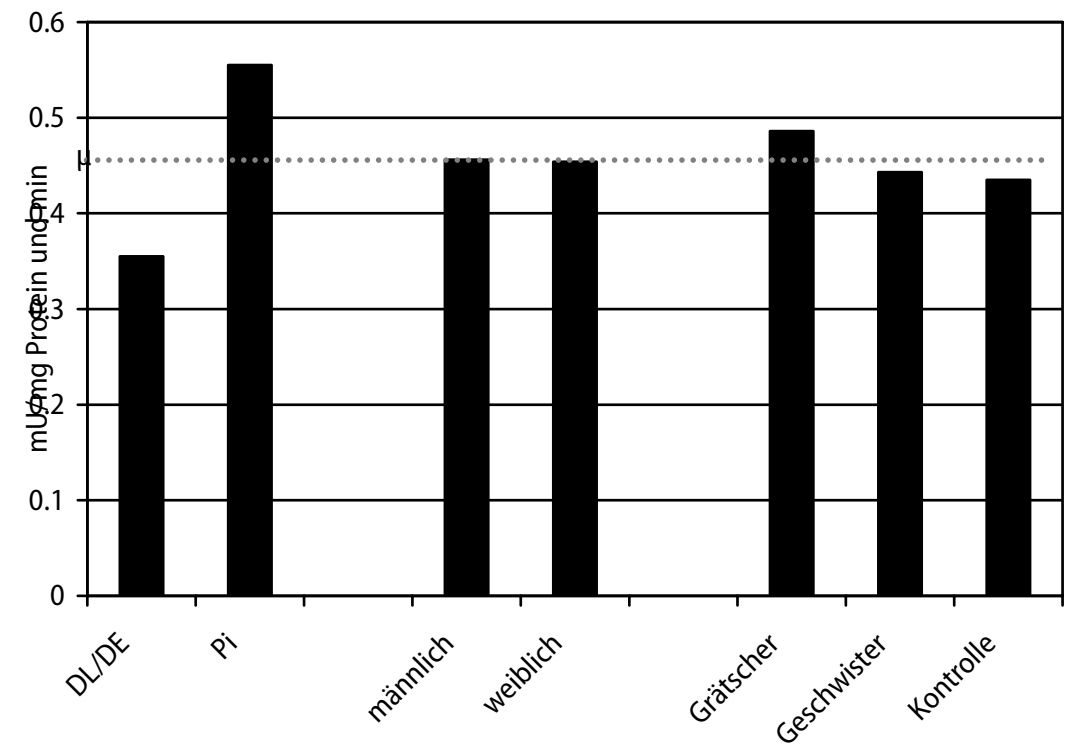

Abbildung 5

Randmittelwerte der CPTI-Aktivität in Muskelmitochondrien von Saugferkeln für die Einflussfaktoren Vaterrasse, Geschlecht und CMH-Gesundheitsstatus unter Angabe des Mittelwertes (0,46 mU/mg und min) Least square mean of the CPT I-activity in muscle mitochondria from piglets for the influence factors father breed, sex and CMH-status including information about the mean $(0,46 \mathrm{mU} / \mathrm{mg}$ and $\mathrm{min})$

\section{Diskussion}

Bei einer stringenten Anwendung der diagnostischen Kriterien nach SCHNAPPERELLE und $\mathrm{KOCH}$ (1980), konnten wesentlich weniger eindeutige »CMH«-Probanden für die Untersuchungen gewonnen werden, als ursprünglich erwartet. Dies ist eine Teilerklärung für die fehlende statistische Absicherung von einigen an sich beachtlichen Merkmalsdifferenzen zwischen Ferkeln mit unterschiedlichem CMH-Gesundheitsstatus.

KERNER et al. (1984) bestimmten bei zwei Tage alten Ferkeln mittels eines radioenzymatischen Tests mit dem Isotop ${ }^{14} \mathrm{C}$ durchschnittliche Gehalte von $359,8 \pm 25 \mathrm{nmol}$ säurelöslichem Gesamtcarnitin und 280,4 $\pm 9,4$ nmol freiem Carnitin pro g Skelettmuskelfrischmasse. Im Vergleich dazu wurden in der vorliegenden Untersuchung bei Umrechnung der gemessenen Gehalte in der Muskeltrockenmasse auf die Frischsubstanz mehr als doppelt so hohe Konzentrationen an freiem und gesamtem Carnitin nachgewiesen. Auch die in dieser Untersuchung ermittelten Acylcarnitingehalte betrugen fast das Doppelte der von KERNER et al. (1984) mittels HPLC gemessenen Werte $(87,1 \pm 5,8 \mathrm{nmol}$ Acylcarnitin/g Frischmasse). Die Unterschiede könnten sich möglicherweise auf das höhere Durchschnittsalter der hier beprobten Ferkel zurückführen lassen (Tabelle 1). Der Anteil am Gesamtcarnitin beträgt sowohl bei KERNER et al. (1984) als auch in der vorliegenden Untersuchung ca. $80 \%$ für das freie Carnitin, bzw. $20 \%$ für das Acylcarnitin.

Vergleicht man die von $\mathrm{CMH}$ betroffenen Ferkel mit den gesunden Tieren, so beobachtet man nicht nur, dass die Grätscher höhere Gesamtcarnitingehalte in der Adduktorenmuskulatur aufweisen, auch die einzelnen Carnitinfraktionen stehen in 
unterschiedlichem Verhältnis zueinander. Auffallend sind hierbei die verhältnismäßig hohen Acylcarnitinkonzentrationen der Grätscherferkel. Die Anhäufung der Carnitinester im Gewebe könnte auf verschiedenen Ursachen beruhen:

FOSTER und HARRIS (1987) führen einen solchen Anstieg in der Muskulatur auf eine verstärkte Muskeltätigkeit zurück. Zwar erscheint bei der CMH die Adduktorenmuskulatur funktionsuntüchtig, dennoch könnten die vermehrten Anstrengungen des Ferkels zur Nahrungs- und Wärmequelle zu gelangen eine verstärkte Belastung der gesamten Muskulatur bewirken. Weiterhin könnte die durch die Nahrungskarenz erzwungene Mobilisierung der spärlichen Fettdepots zur Energiegewinnung und Wärmeproduktion durch Kältezittern den Acylcarnitingehalt erhöhen. Nicht mit dem weiteren spontanen Ausheilen der $\mathrm{CMH}$ zu vereinbaren wäre eine Akkumulation von Acylestern aufgrund einer Insuffizienz der renalen Eliminierung.

Die wichtigste Carnitinquelle stellt für das Ferkel die Muttermilch dar. Nun wird gerade beim Schwein im Gegensatz zu anderen Haustierarten und dem Menschen dem Jungtier mit der Milch hauptsächlich verestertes Carnitin zugeführt. Es wäre aber nicht erklärlich, warum gerade die schwächeren, von Nahrungsmangel bedrohten Grätscher mehr Milch aufnehmen sollten als gesunde Ferkel.

Demgegenüber konnte mit der vorliegenden Untersuchung nachgewiesen werden, dass die Gehalte der jeweiligen Carnitinfraktion im Blutplasma der Ferkel in einem engen positiv gerichteten Zusammenhang mit den jeweiligen Anteilen im Plasma der Muttersau stehen. Das Ferkel kommt mit geringen Plasmacarnitingehalten zur Welt (BIEBER et al. 1973). KAISER (1997) beobachtete eine Erhöhung der Plasmakonzentration bei Saugferkeln als Folge erhöhter Carnitingehalte in der Sauenmilch. Mögliche Ursachen für die hochkorrelierten Carnitingehalte zwischen Sau und Ferkel könnten des Weiteren eine vererbte Leistungsfähigkeit der Carnitinbiosynthese oder aber die von NOVAK et al. (1981) beobachtete maternale transplazentare Übertragung von freiem Carnitin sein.

Die in dieser Untersuchung im Blutplasma gemessenen Gehalte an freiem und gesamtem Carnitin lagen um das dreifache höher als die von KERNER et al. (1984) angegebenen Werte. Die von den genannten Autoren gemessenen Acylcarnitinkonzentrationen betrugen die Hälfte der in der vorliegenden Untersuchung ermittelten. Übereinstimmungen konnten in den berechneten prozentualen Anteilen von ca. $66 \%$ freiem Carnitin und ca. 34\% Acylcarnitin am Gesamtcarnitin festgestellt werden. Die an $\mathrm{CMH}$ erkrankten Ferkel wiesen im Gegensatz zu den Verhältnissen in der Skelettmuskulatur einen tendenziell niedrigeren Acylcarnitinanteil im Blutplasma auf als die Gesunden. Vergleicht man die Carnitingehalte im Plasma und der Muskulatur der Ferkel, so ist anhand der berechneten Korrelationen der absoluten Werte keine nennenswerte Beziehung zu erkennen. REBOUCHE (1989) ermittelte zwar eine signifikante positive Korrelation zwischen den Carnitingehalten in der Skelettmuskulatur und dem Blutplasma bei der Ratte, konnte diese aber nur für die Plasmacarnitingehalte im Normalbereich bestätigen. Die Untersuchungen von LENNON et al. (1986) und COSTELL et al. (1989) unterstützen dagegen die Beobachtung, dass die Bestimmung des Plasmacarnitingehaltes keine Aussage über den Carnitingehalt in der Skelettmuskulatur erlaubt.

Des Weiteren wurde der Carnitingehalt in den Lymphozyten des Blutes untersucht, da diese, sofern eine Vergleichbarkeit mit den Verhältnissen in der Muskulatur nachzuweisen 
wäre, für diagnostische Zwecke leichter zu gewinnen wären als ein Muskelbioptat. Während in der Skelettmuskulatur und im Plasma das freie Carnitin den größten Anteil am Gesamtcarnitin einnimmt, herrscht in den Lymphozyten das Acylcarnitin vor. DEUFEL (1981) weist Acylcarnitingehalte um die $75 \%$ vom Gesamtcarnitin in den Lymphozyten des Menschen nach. Vom Schwein liegen bisher keine Vergleichswerte vor. Trotz des umgekehrten Verhältnisses von freiem Carnitin zu Acylcarnitin sind bei den Grätschern wiederum die höchsten Acylcarnitingehalte zu beobachten. Dennoch bleibt fraglich, ob das Hinzuziehen der Carnitinfraktionsgehalte in den Lymphozyten einen pathognomonischen Hinweis auf die CMH liefern kann. Zwar wurden signifikant hohe positive Korrelationen zwischen dem freien bzw. gesamten Carnitin in Lymphozyten und M. adduktor berechnet, diese Beziehung konnte aber ausgerechnet für die Schlüsselfraktion des Acylcarnitins nicht nachgewiesen werden, so dass die Blutprobe nicht als sicherer Ersatz für eine Muskelprobe in Betracht zu ziehen ist.

Mit der Untersuchung des ATP-Bildungsvermögens der Lymphozyten- und Skelettmuskelmitochondrien wurde eine weitere mögliche Beeinträchtigung des Stoffwechsels bei an CMH erkrankten Ferkeln in Augenschein genommen. Während in den Lymphozyten unabhängig vom eingesetzten Substrat stets eine tendenzielle Überlegenheit in der ATP-Synthese bei Ferkeln aus gesunden Würfen zu beobachten war, waren die Muskelmitochondrien von Ferkeln aus von CMH betroffenen Würfen besonders beim Einsatz des Substrats Palmitoylcarnitin in der ATP-Produktion schwächer, das Succinat konnte dagegen deutlich besser verwertet werden. Es liegt der Verdacht nahe, dass Grätscherferkel und ihre Geschwister Probleme bei der energetischen Verwertung langkettiger Fettsäuren aufweisen könnten.

Die CPT I ist eine wichtige Schaltstelle in der Regulation des Fettsäurenstoffwechsels. KERNER et al. (1984) beobachteten einen Anstieg der Aktivität dieses Enzyms im Skelettmuskel von Ferkeln vom Tag der Geburt bis zum zweiten Lebenstag von 0,02 U/g Frischsubstanz auf $0,07 \mathrm{U} / \mathrm{g}$ Frischsubstanz. Unter Berücksichtigung eines theoretischen Proteingehaltes von $16 \%$ im Fleisch stimmen diese Werte mit denen aus der vorliegenden Untersuchung überein. Beim Vergleich der Enzymaktivität von betroffenen und gesunden Würfen konnten keine Unterschiede festgestellt werden, so dass ein CPT IMangel als ursächlich für die durch CMH bedingten Störungen ausscheidet.

\section{Danksagung}

Teile dieser Untersuchung wurden dankenswerter Weise durch eine Forschungsspende der H. Wilhelm Schaumann Stiftung, Hamburg unterstützt.

\section{Literatur}

Bergmeyer HU (Hrsg) (1974) Methoden der enzymatischen Analyse. Bd I, II. Verlag Chemie GmbH Weinheim

Bieber LL, Markwell MAK, Blair M, Helmrath TA (1973) Studies on the development of carnitine palmytoyltransferase and fatty acid oxidation in liver mitochondria of neonatal pigs. Biochem Biophys Acta 326, 145-54

Bölcskei A, Bilkei G, Biro O, Clavadetscher E, Goos T, Stelzer P, Bilkei H, Wegmüller S (1996) Der Einfluss des Zeitpunktes der Partusinduktion auf das Auftreten der kongenitalen myofibrillären Hypoplasie Kurzbericht aus der Praxis. Dtsch Tierärztl Wschr 103, 21-2 
Bremer J (1981) The effect of fasting on the activity of liver carnitine palmitoyltransferase and its inhibition by malonyl CoA. Biochim Biophys Acta 665, 628-31

Carpentieri U, Sordahl LA (1980) Respiratory and calcium transport functions of mitochondria isolated from normal and transformed human lymphocytes. Cancer Research 40, 221-4

Costell M, O'Connor JE, Grisolia S (1989) Age-dependent decrease of carnitine content in muscle of mice and humans. Biochem Biophys Res Comm 161, 1135-43

Deufel T (1981) Klinisch-biochemische Untersuchung zum Carnitinstoffwechsel des Menschen und zur Diagnostik von Carnitinmangel-Syndromen. Diss med vet München

Ducatelle R, Maenhout D, Coussement W, Hoorens JK (1986) Spontaneus and experimental myofibrillar hypoplasia and its relation to splayleg in newborn pigs. J Comp Path 96, 433-44

Foster CVL, Harris RC (1987) Formation of acetylcarnitine in muscle of horse during high intensity exercise. Eur J Appl Phys 56, 639-42

Freitag M, Klein D (2003) Den Erbfehlern auf der Spur. SUS 4, 36-9

Friedmann S, Fraenkel G (1955) Reversible enzymatic acetylation of carnitine. Arch Biochem Biophys 59, 491-501

Fritz IB (1955) The effects of muscle extracts on the oxidation of palmitic acid by liver slices and homogenates. Acta Physiol Scand 34, 367-85

Gustavsen HSM (2000) Bestimmung des L-Carnitingehaltes in rohen und zubereiteten pflanzlichen und tierischen Lebensmitteln. Diss med vet Hannover

Jirmanova I, Lojda L (1985) Dexamethasone applied to pregnant minisows induces splayleg in minipiglets. Zbl Vet Med A 32, 445-58

Kaiser U (1997) Einfluss einer L-Carnitinzulage im Futter von hochtragenden und säugenden Sauen auf die Aufzuchtleistung und den Carnitinstatus bei Sauen und Ferkeln. Diss med vet Tierärztl Hochschule Hannover

Kerner J, Bieber LL (1983) A radioisotopic-exchange method for quantitation of short-chain (acidsoluble) acylcarnitines. Analytical Biochem 134, 459-66

Kerner J, Froseth JA, Miller ER, Bieber LL (1984) A study of the acylcarnitine content of sows colostrum milk and newborn piglet tissues: Demonstration of high amounts of isovalerylcarnitine in colostrum and milk. J Nutr 114, 854-61

Lennon DLF, Shrago ER, Madden M, Nagle FJ, Hanson P (1986) Dietary carnitine intake related to skeletal muscle and plasma carnitine concentrations in adult men and women. Am J Clin Nutr 43, 234-8

Lowry OH, Rosebrough NJ, Farr AL, Randall RJ (1951) Protein measurement with the folin phenol reagent. J Biol Chem 193, 265-7

Maak S, Jaesert S, Neumann K, Yerle M, von Lengerken G (2001) Isolation of expressend sequence tags of skeletal muscle of neonatal healthy and splayleg piglets and mapping by somatic cell hybrid analysis. Anim Gen 32, 303-7

Maak S, Jaesert S, Neumann K, von Lengerken G (2003) Characterisation of the porcine CDKN3 gene as a potential candidate for congenital splayleg in piglets. Gen Sel Evol 35 Suppl 1, S157-65

Maak S, Wicke M, Swalve HH (2005) Analysis of gene expression in specific muscles of swine and turkey. Arch Tierz 48 SI, 135-40

Maak S, Neumann K, Swalve HH (2007) Polymorhic putative regulatory elements at the MYF5/MYF6 loci and expression analysis in neonatal pigs. Arch Tierz $50 \mathrm{SI}, 44-8$

McGarry JD, Foster DW In H Bergmeyer (Hrsg) (1985) Methoden der enzymatischen Analyse. 3. Aufl Bd 7 Verlag Chemie Weinheim, 1806-19

Mersmann HJ, Goodman J, Houk JM, Anderson S (1972) Studies of the biochemistry of mitochondria and cell morphology in the neonatal pig. J Cell Biol 53, 335-47

Norum KR (1964) PalmitylCoA Carnitine palmityltransferase. Purification from calf-liver mitochondria and some properties of the enzyme. Biochim Biophys Acta 89, 95

Novak M, Monkus EF, Chung D, Buch D (1981) Carnitine in the perinatal metabolism of lipids. I. Relationship between maternal and fetal plasma levels of carnitine and acylcarnitine. Pediatrics 67, 95-100

Rebouche CJ (1989) Carnitine transport and tissue carnitine accretion in rats. Biochem Biophys Acta $1033,111-3$

Schmidt I, Herpin P (1998) Carnitine Palmitoyltransferase I (CPT I) activity and its regulation by MalonylCoA are modulated by age and cold exposure in skeletal muscle mitochondria from newborn pigs. J Nutr 128, 886-93 
Schnapperelle H, Koch J (1980) Untersuchungen zum Auftreten und zur Therapie des angeborenen Beinspreizens bei Saugferkeln Mh Vet-Med 35, 858-60

Schwarz S (2003) Kartierung von Loci für kongenitales Ausgrätschen beim neugeborenen Ferkel. Diss agr Universität Kiel

Sellier P, Olliver L (1982) Genetics study of splayleg in the new-born piglet. I. Multifactorial model with one threshold. Ann Genet Selec Anim 14, 77-92 [in Französisch]

Sellier P, Dando E, Dando P (1999) Induction of parturition in the sow and incidence of splayleg syndrome in the newborn piglet. Ann Zootech 48, 153-61

Stigler J, Distl O, Kruff B, Kräußlich H (1992) Zur Erblichkeit wirtschaftlich relevanter Mißbildungen beim Schwein. Tierärztl Umschau 47, 883-6

Tucek S, Svoboda T, Ricny J (1985) The concentration of cholin and the activities of cholinesterase creatine kinase and LDH in the blood plasma of piglets with the syndrom splayleg. Zbl Vet Med A 32, $1-10$

Vanyi A, Bata A, Glavitts R, Kovacs F (1994) Perinatal östrogen syndrom in swine. Acta Vet Hungaria 42, 433-46

Vogt DW, Gipson TA, Akremi B, Dover S, Ellersieck MR (1984) Associations of sire breed birth weight and sex in pigs with congenital splayleg. Am J Vet Res 45, 2408-9

Ward PS (1978) The splayleg syndrome in newborn pigs. Vet Bulletin 48/4 I, 279-294; II 381-99

Received 15 May 2008, accepted 17 November 2008.

Corresponding author:

Prof. Dr. VLADO DZAPO

email: vladimir.dzapo@agrar.uni-giessen.de

Department of Animal Breeding and Genetics, Faculty 09 - Agricultural Sciences, Nutritional Sciences and Environmental Management, Justus-Liebig-Universität Gießen, Oberer Hardthof 18, 35398 Gießen, Germany 